\title{
Identifying Native Point Defects in the Topological Insulator $\mathrm{Bi}_{2} \mathrm{Te}_{3}$
}

\author{
Asteriona-Maria Netsou, ${ }^{*, \dagger}$ Dmitry A. Muzychenko, ${ }^{*, \dagger}$ Heleen Dausy, ${ }^{\dagger}$ Taishi \\ Chen, " Fengqi Song, ${ }^{\S}$ Koen Schouteden," Margriet J. Van Bael, ${ }^{\dagger}$ and Chris Van \\ Haesendonck $^{\dagger}$
}

$†$ †uantum Solid State Physics (QSP), KU Leuven, BE-3001 Leuven, Belgium $\ddagger$ Faculty of Physics, Lomonosov Moscow State University, 119991 Moscow, Russia

IInstitute for Solid State Physics, University of Tokyo, Kashiwa 27r-8581, Japan $\S$ National Laboratory of Solid State Microstructures, Collaborative Innovation Center of Advanced Microstructures, and Department of Physics, Nanjing University, Nanjing 210093, China

||Laboratory for Semiconductor Physics, KU Leuven, BE-3001 Leuven, Belgium

E-mail: asterionamaria.netsou@kuleuven.be; mda@spmlab.ru

\begin{abstract}
We successfully identified native point defects that occur in $\mathrm{Bi}_{2} \mathrm{Te}_{3}$ crystals by combining high-resolution bias-dependent scanning tunneling microscopy and density functional theory based calculations. As-grown $\mathrm{Bi}_{2} \mathrm{Te}_{3}$ crystals contain vacancies, antisites and interstitial defects that may result in bulk conductivity and therefore may change the insulating bulk character. Here, we demonstrate the interplay between the growth conditions and the density of different types of native near-surface defects. In particular, scanning tunneling spectroscopy reveals the dependence on not only the local atomic environment, but also on the growth kinetics and the resulting sample doping
\end{abstract}


from $n$-type towards intrinsic crystals with the Fermi level positioned inside the energy gap. Our results establish a bias-dependent STM signature of the $\mathrm{Bi}_{2} \mathrm{Te}_{3}$ native defects and shed light on the link between the native defects and the electronic properties of $\mathrm{Bi}_{2} \mathrm{Te}_{3}$, which is relevant for the synthesis of topological insulator materials and the related functional properties.

\section{Keywords}

topological insulators, $\mathrm{Bi}_{2} \mathrm{Te}_{3}$, native point defects, scanning tunneling microscopy, density functional theory

Topological insulators (TIs) represent one of the most active areas of quantum technology related research in condensed matter physics the last couple of years. The intense interest in TI materials soon after their theoretical prediction and experimental confirmation is justified as they realize a new electronic phase with promising applications ranging from advanced electronic and spintronic devices to quantum computing. The topological surface states (TSSs) of a three-dimensional TI provide robust conduction on the surface while the bulk is insulating. The low-energy electronic properties of these TSSs are dominated by massless Dirac fermion excitations, where the emerging Dirac cone defines the energy dispersion relations. Furthermore, a spin-momentum locking property is acquired due to spin-orbit interaction and time reversal symmetry that is present in these materials. As a result, backscattering from non-magnetic impurities is not allowed in TIs. ${ }^{111}$ However, these fascinating properties experience many challenges caused by intrinsic point defects that exist naturally in binary tetradymites, including $\mathrm{Bi}_{2} \mathrm{Te}_{3}$ and $\mathrm{Bi}_{2} \mathrm{Se}_{3} \cdot \frac{12}{} \mathrm{Bi}$ antisite defects denoted as $\mathrm{Bi}_{\mathrm{Te}}$, Te antisite defects denoted as $\mathrm{Te}_{\mathrm{Bi}}$, and $\mathrm{Te}$ vacancies denoted as $\mathrm{V}_{\mathrm{Te}}$, are native defects with low formation energies that dope $\mathrm{Bi}_{2} \mathrm{Te}_{3}$ crystals, which in turn exhibit bulk conductance that overshadows the TSSs. $13+16$ On the other hand, identifying and investigating the impact of such native point defects close to the surface of the $\mathrm{Bi}_{2} \mathrm{Te}_{3}$ is critical for improving the sample quality and the TI electronic properties necessary for TI-based 
devices.

$\mathrm{Bi}_{2} \mathrm{Te}_{3}$ is a well-studied material due to its thermoelectric properties and it was already understood many decades ago that Te concentration during synthesis affects the sign of the charge carriers. Crystals grown under stoichiometric conditions turn out to be doped samples with a $\mathrm{Bi}$ excess. To achieve charge neutrality an excess of Te exceeding the $\mathrm{Bi}_{2} \mathrm{Te}_{3}$ stoichiometric ratio for the material synthesis is needed. The very early experiments revealed that $p$-type $\mathrm{Bi}_{2} \mathrm{Te}_{3}$ crystals are formed when there is a higher excess of Te. $\frac{1718}{1 n}$ a more recent experimental study it was found that Te shows a high evaporation tendency during crystal growth and that around 60 at.\% of Te induces $n$-type doping. ${ }^{19}$ To date, the doping scheme remains a rather complicated problem and requires additional experimental results to be better understood.

The unit cell of $\mathrm{Bi}_{2} \mathrm{Te}_{3}$ is rhombohedral and consists of repetitive slabs of five atomic layers (Te1-Bi-Te2-Bi-Te1; see Figure 1), which are referred to as quintuple layers (QLs). For convenience, the crystal structure of $\mathrm{Bi}_{2} \mathrm{Te}_{3}$ is commonly described using a hexagonal unit cell that consists of 3 QLs. The QLs are weakly bonded by van der Waals forces occurring between neighboring Te1 sublattices, while strong interatomic bonding inside the QLs is of covalent and ionic nature. $20-22$ The weak van der Waals forces allow the in situ cleaving of the $\mathrm{Bi}_{2} \mathrm{Te}_{3}$ layered material to preferably reveal a Te1 terminated surface. It has been calculated based on density functional theory (DFT) that antisite and vacancy defects created in the Te1 sublattice have lower formation energies than analogous defects created in the Te2 sublattice. Furthermore, antisite defects should be the most dominant with $\mathrm{Bi}_{\mathrm{Te}}$ dominating for Bi-rich conditions and $\mathrm{Te}_{\mathrm{Bi}}$ dominating for Te-rich conditions. Both antisite defects have similar probability to exist in the material with the exact stoichiometric composition. The majority of the DFT-based calculations agree that the defects with the next lower formation energy after antisite defects, especially for Bi-rich conditions, are vacancies on the Te1 sublattice. $\frac{12[16[23}{25]} \mathrm{Te}_{\mathrm{Bi}}$ antisite defects act as single donors and $\mathrm{Bi}_{\mathrm{Te}}$ antisite defects act as single acceptors. On the other hand, vacancies are strong perturbations with $\mathrm{V}_{\mathrm{Te}}$ acting 
as double donors and $\mathrm{V}_{\mathrm{Bi}}$ as triple acceptors. $\frac{1226}{226}$ However, it is noteworthy that different DFT-based calculations have shown that $\mathrm{V}_{\mathrm{Te}}$ defects are charge neutral. $\underline{23}$

Even though $\mathrm{Bi}_{2} \mathrm{Te}_{3}$ has been widely investigated during the past few years due to its TI properties, there is a lack of experimental studies focusing on the native point defects to support the first-principles calculations of defect formation and characterization. Advances in high-resolution scanning transmission electron microscopy (HR-STEM) have made it possible for this technique to provide structural and chemical information down to the atomic scale about defects in tetradymite crystals and thin films. Up to date this information remains restricted to defects located below the top surface QLs. $\frac{27 \mid 28}{28}$ On the other hand, scanning tunneling microscopy (STM) and spectroscopy (STS) allow direct visualization of the atomicscale surface topography and investigation of the two-dimensional (2D) TSSs that appear within the top surface QLs. ${ }^{23|29| 30}$ For conclusively identifying the origin of the native defects in $\mathrm{Bi}_{2} \mathrm{Te}_{3}$, a direct comparison between high-resolution STM images and simulated STM images is required.

Here, we present a comprehensive analysis of the identity of the native defects as well as their impact on the electronic properties of $\mathrm{Bi}_{2} \mathrm{Te}_{3}$, by means of high-resolution STM/STS combined with DFT-based calculations. In particular, we successfully identify (1) $\mathrm{V}_{\mathrm{Te}}$ vacancy defects and $\mathrm{Bi}_{\mathrm{Te}}$ antisite defects on the Te1 sublattice and on the Te2 sublattice, (2) $\mathrm{Te}_{\mathrm{Bi}}$ antisite defects occurring on the Bi sublattice (for the $2^{\text {nd }}$ atomic layer of the $\mathrm{QL}$ ), (3) Bi interstitial defects in the van der Waals gap of the QLs, and (4) Bi adatoms on the surface of $\mathrm{Bi}_{2} \mathrm{Te}_{3}$. The considered single crystals are (A) $\mathrm{Bi}_{2} \mathrm{Te}_{3}$ grown under Te-rich conditions (starting from a mixture with a Te excess of about 0.1 at.\% Te), (B) $\mathrm{Bi}_{2} \mathrm{Te}_{3}$ grown under the same Te-rich conditions, but with a slow cooling (SC) rate (grown with a three times slower cooling rate than the Te-rich single crystal (A)), and (C) $\mathrm{Bi}_{2} \mathrm{Te}_{3}$ grown starting from a stoichiometric mixture with exactly 40 at.\% of Bi and 60 at.\% of Te. We further on more briefly refer to these single crystals as "Te-rich" $\mathrm{Bi}_{2} \mathrm{Te}_{3}$, "Te-rich $\mathrm{SC}$ " $\mathrm{Bi}_{2} \mathrm{Te}_{3}$ and "stoichiometric" $\mathrm{Bi}_{2} \mathrm{Te}_{3}$, respectively. We determine the densities of the native defects for 
each sample to reveal the complexity of the doping mechanism and the importance of not only the thermodynamics but also the growth kinetics. We demonstrate by STS measurements that the Te-rich $\mathrm{Bi}_{2} \mathrm{Te}_{3}$ crystals approach intrinsic electronic behavior with the Fermi level positioned inside the energy gap. Furthermore, for the Te-rich growth conditions we demonstrate the existence of Bi adatoms, for which STS data reveal the presence of an electronic resonance above the Fermi level. Our results fill the gap resulting from the absence of sufficient experimental data on the naturally occurring point defects in $\mathrm{Bi}_{2} \mathrm{Te}_{3}$ and can direct future synthesis to improve the functional properties of TI materials.

(a)

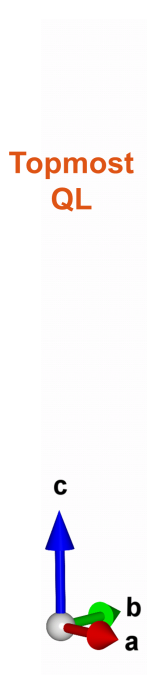

Side view

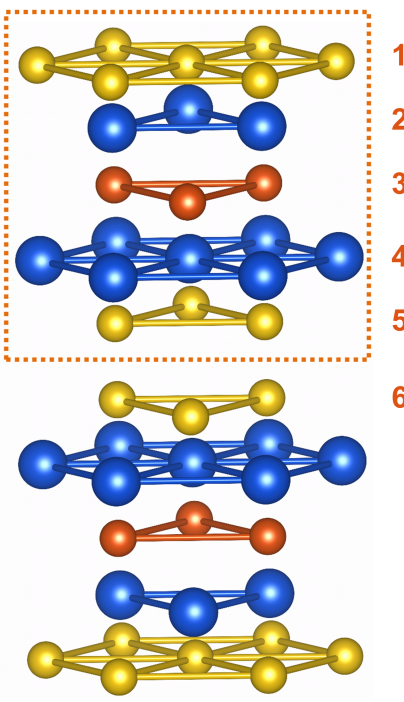

(b) Top view

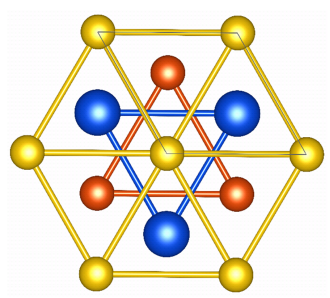

Figure 1: Crystal structure of $\mathbf{B i}_{\mathbf{2}} \mathbf{T} \mathbf{T e}_{\mathbf{3}}$. (a) Side view of the crystal structure showing two QLs. Orange dotted rectangle presents the topmost QL and the lateral numbers indicate the atomic layers of the Te1 terminated surface. (b) Top view of the crystal structure along the $z$-direction.

\section{Results and discussion}

The atomically resolved STM topography of the surface of the Te-rich $\mathrm{Bi}_{2} \mathrm{Te}_{3}$ crystal after in situ cleavage is presented in Figure $2 \mathrm{a}$. Figures $2 \mathrm{~b}$ and $2 \mathrm{c}$ present the corresponding high-resolution STM images of the surface topography of the Te-rich $\mathrm{SC} \mathrm{Bi}_{2} \mathrm{Te}_{3}$ and the stoichiometric $\mathrm{Bi}_{2} \mathrm{Te}_{3}$, respectively. The STM topographies reveal the existence of native 
defects, whose type and density differs depending on the growth conditions. Native defects appear as protrusions or depressions, most of them of trigonal geometry, which is due to the chemical bonding in the $\mathrm{Bi}_{2} \mathrm{Te}_{3}$ crystals. $\frac{2122}{2}$ For an applied negative bias in Figures 2 a-c, features appearing as bright protrusions correspond to defects that are electron acceptors and features appearing as dark depressions reflect the presence of defects that are electron donors, while at positive bias the opposite contrast will appear. 23129

In order to identify the type and the exact position of the native defects in the QL, DFTbased calculations are employed. The comparison between the experimental STM images and the simulated STM images is presented in Figures $2 \mathrm{~d}-\mathrm{g}$, where the bias voltages are selected to highlight the most apparent topographic appearance for each defect type (for experimental STM images of the native defects within a broader bias voltage range see Figures SM1, SM2 and $\mathrm{SM} 3) . \mathrm{Bi}_{\mathrm{Te}}$ antisite defects occurring in the $1^{\mathrm{st}}, 3^{\mathrm{rd}}$ and $5^{\text {th }}$ atomic layer of the topmost QL and in the $1^{\text {st }}$ atomic layer of the next QL (6 $6^{\text {th }}$ atomic layer from the top) are presented in Figure 2d, for both filled (negative bias) and empty (positive bias) states of the experimental and simulated STM images. Figure 22 illustrates the $\mathrm{Te}_{\mathrm{Bi}}$ antisite defect occurring in the $2^{\text {nd }}$ atomic layer and Figure 2 f illustrates the Te vacancies occuring in the $1^{\text {st }}, 3^{\text {rd }}$ and $5^{\text {th }}$ atomic layer. The Bi interstitial defect in the van der Waals gaps between QLs is clearly resolved as well in the experimental STM images, observed in this case in between the $5^{\text {th }}$ and $6^{\text {th }}$ atomic layer (denoted as $\mathrm{Bi}_{\mathrm{i}}, 5-6$ in Figure $2 \mathrm{~s}$ ) from the top surface. Furthermore, our experiments reveal the existence of $\mathrm{Bi}$ adatoms measured for the Te-rich and the Te-rich $\mathrm{SC} \mathrm{Bi}_{2} \mathrm{Te}_{3}$ crystals. Bi adatoms can be observed in Figure $2 \mathrm{~b}$ (denoted as $\mathrm{Bi}_{\mathrm{a}}$ ) and are unambiguously distinct from the $\mathrm{Bi}_{\mathrm{Te}}, 1$ defects due to their observed mobility on the surface (see Figure SM5). From our experimental data, two possible mechanisms for the formation of the Bi adatom defects can be put forward. The first mechanism assumes that they correspond to the naturally trapped excess Bi atoms in the van der Waals gaps and are revealed after cleaving. The second mechanism assumes that they result from imperfect cleaving. The assignment of the observed adatoms as $\mathrm{Bi}$ adatoms is motivated by the presence of $\mathrm{Bi}_{\mathrm{i}}, 5-6$ 
(a) Te-rich $\mathrm{Bi}_{2} \mathrm{Te}_{3}$

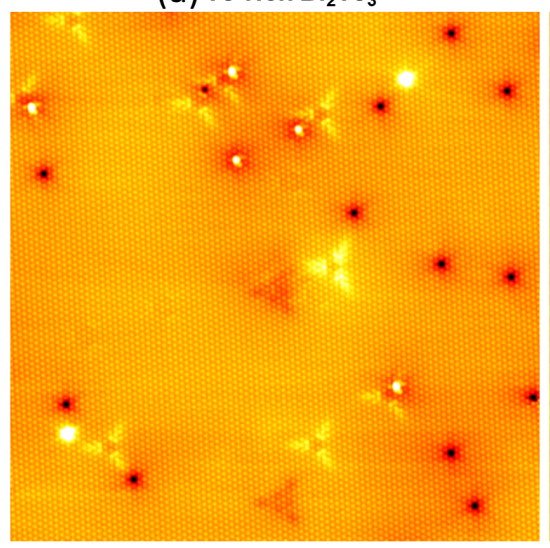

(d) STM Filled states DFT STM E

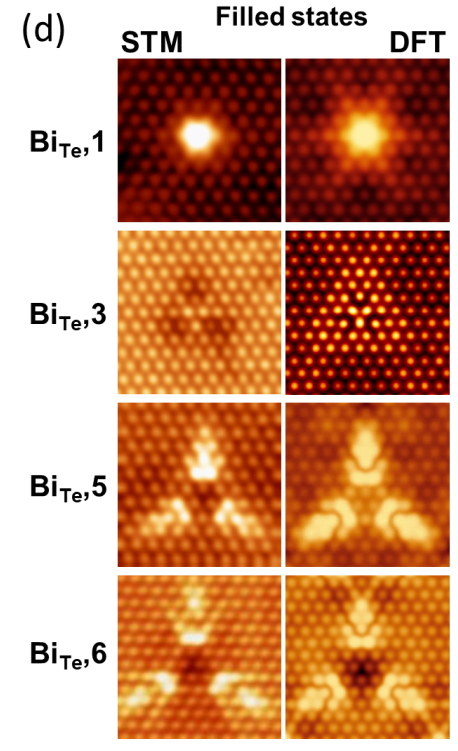

(e) STM Filled states (b) Te-rich SC $\mathrm{Bi}_{2} \mathrm{Te}_{3}$

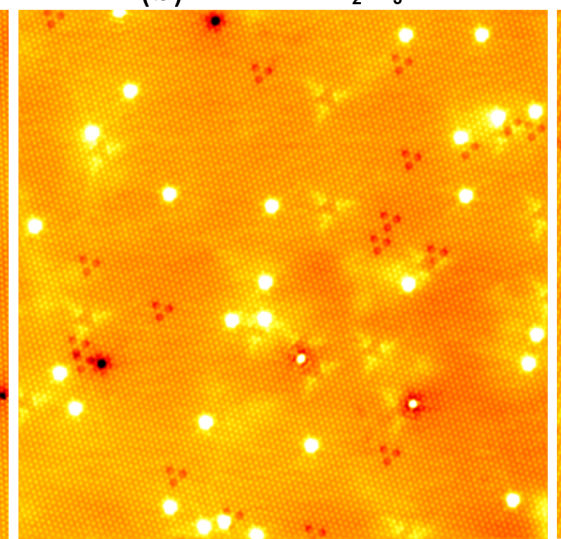

(c) Stoichiometric $\mathrm{Bi}_{2} \mathrm{Te}_{3}$

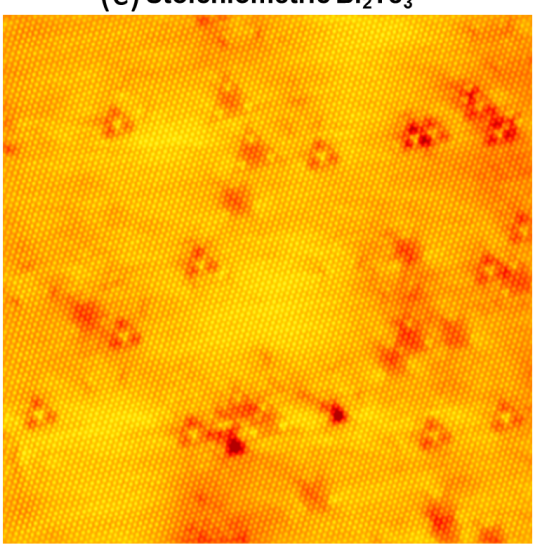

(f) STM Filled states DFT STM Empty states

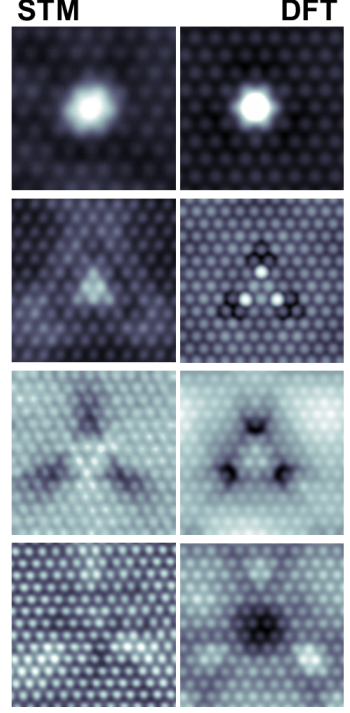

Empty states

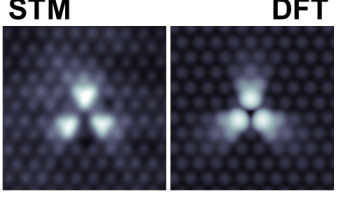

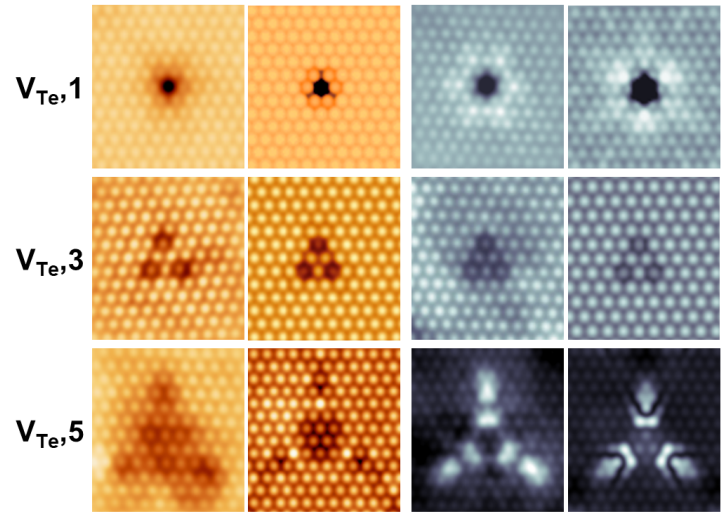

(g) STM Filled states DFT STM ${ }_{\text {DFT }}^{\text {Empty states }}$
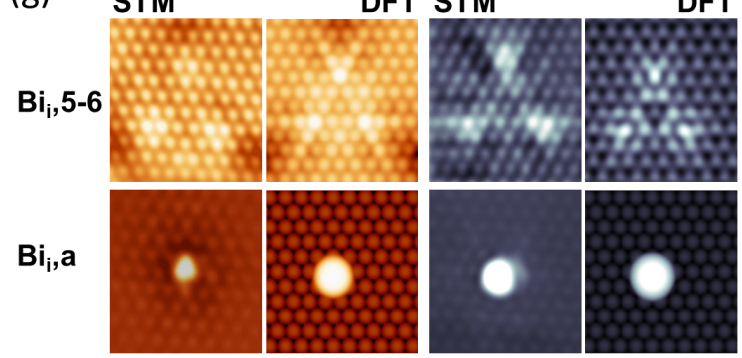

Figure 2: Bias-dependent STM signatures of native point defects in the vicinity of the $\mathbf{B i}_{\mathbf{2}} \mathbf{T e}_{\mathbf{3}}$ surface. Typical atomically resolved $40 \times 40 \mathrm{~nm}^{2}$ filled-states STM topographies of (a) Te-rich $\mathrm{Bi}_{2} \mathrm{Te}_{3}(V=-0.5 \mathrm{~V})$, (b) Te-rich $\mathrm{SC} \mathrm{Bi}_{2} \mathrm{Te}_{3}(V=-0.7 \mathrm{~V})$, (c) Stoichiometric $\mathrm{Bi}_{2} \mathrm{Te}_{3}(V=-0.4 \mathrm{~V})$. Color height scales are (a) 1.9, (b) 1.74, and (c) $0.22 \AA$. Experimental and simulated STM images for both filled (orange-brown color) and empty (gray-blue color) states for (d) $\mathrm{Bi}_{\mathrm{Te}}$ defects located in the $1^{\text {st }}\left(\mathrm{Bi}_{\mathrm{Te}}, 1\right.$ at $\left.V= \pm 0.5 \mathrm{~V}\right)$, $3^{\text {rd }}\left(\mathrm{Bi}_{\mathrm{Te}}, 3\right.$ at $\left.V= \pm 0.5 \mathrm{~V}\right), 5^{\text {th }}\left(\mathrm{Bi}_{\mathrm{Te}}, 5\right.$ at $\left.V= \pm 0.5 \mathrm{~V}\right)$ and $6^{\text {th }}\left(\mathrm{Bi}_{\mathrm{Te}}, 6\right.$ at $\left.V= \pm 0.7 \mathrm{~V}\right)$ atomic layer, (e) $\mathrm{Te}_{\mathrm{Bi}}$ defects located in the $2^{\text {nd }}\left(\mathrm{Te}_{\mathrm{Bi}}, 2\right.$ at $V=-0.5 \mathrm{~V}$ and $\left.V=0.4 \mathrm{~V}\right)$ atomic layer, (f) $\mathrm{V}_{\mathrm{Te}}$ defects located in the $1^{\text {st }}\left(\mathrm{V}_{\mathrm{Te}}, 1\right.$ at $V=-0.5 \mathrm{~V}$ and $\left.V=0.7 \mathrm{~V}\right), 3^{\text {rd }}$ $\left(\mathrm{V}_{\mathrm{Te}}, 3\right.$ at $V=-0.7 \mathrm{~V}$ and $\left.V=0.3 \mathrm{~V}\right)$ and $5^{\text {th }}\left(\mathrm{V}_{\mathrm{Te}}, 5\right.$ at $V=-0.7 \mathrm{~V}$ and $\left.V=0.5 \mathrm{~V}\right)$ atomic layer and $(\mathrm{g}) \mathrm{Bi}_{\mathrm{i}}$ defects located in between the $5^{\text {th }}$ and $6^{\text {th }}\left(\mathrm{Bi}_{\mathrm{i}}, 5-6\right.$ at $\left.V= \pm 0.1 \mathrm{~V}\right)$ atomic layer and on top of the $1^{\mathrm{st}}\left(\mathrm{Bi}_{\mathrm{a}}\right.$ at $V=-0.5 \mathrm{~V}$ and $\left.V=0.7 \mathrm{~V}\right)$ atomic layer. 
defects, corresponding to interstitial $\mathrm{Bi}$ atoms in the van der Waals gap in between the top and second QLs. Bi interstitial defects in the van der Waals gaps of $\mathrm{Bi}_{2} \mathrm{Se}_{3}$ crystals have also been identified by HR-STEM measurements. ${ }^{28}$ A reasonable assumption is to assign the adatoms as Bi adatoms that are formed according to the first mechanism mentioned above. However, experimental and simulated STM images of the adatoms cannot unequivocally distinguish between $\mathrm{Bi}$ and Te adatoms, implying the possibility that the adatoms are Te adatoms cannot be excluded. The good agreement between experimental results and DFTbased calculations for both filled and empty states, makes the identification of the commonly observed $\mathrm{Bi}_{2} \mathrm{Te}_{3}$ native defects possible. Therefore, Figures $2 \mathrm{~d}$-g can serve as bias-dependent STM signatures for the most frequent native defects near the surface of cleaved $\mathrm{Bi}_{2} \mathrm{Te}_{3}$.

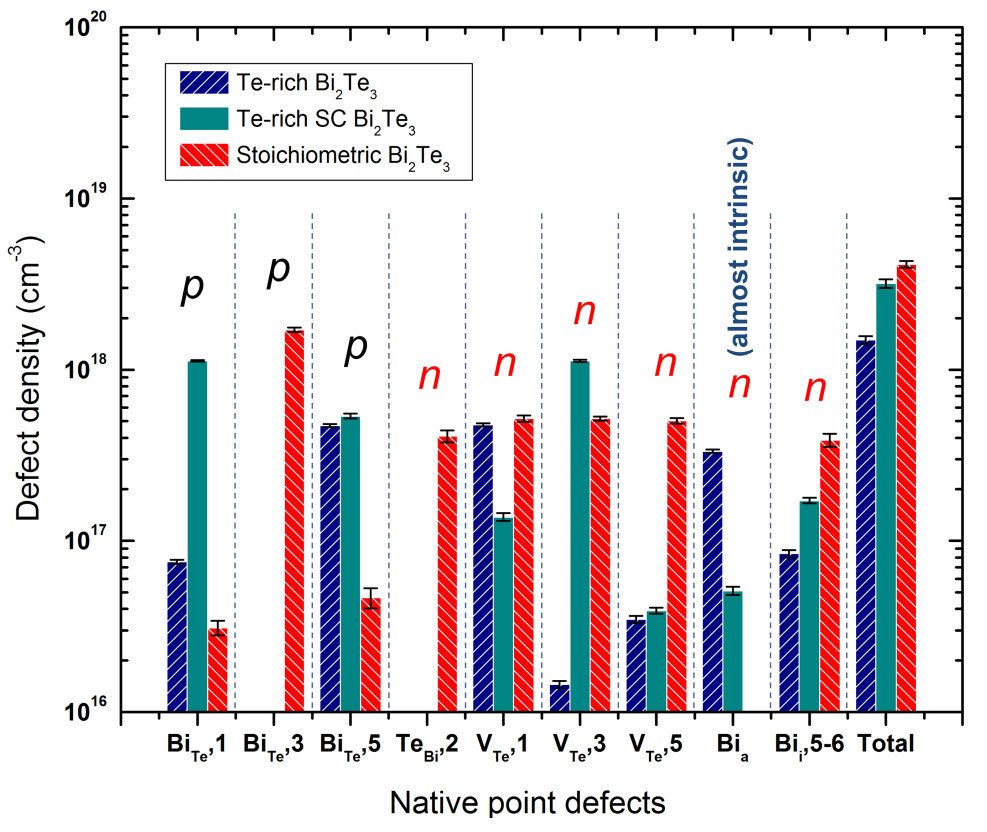

Figure 3: Density of native point defects in the vicinity of the $\mathrm{Bi}_{2} \mathrm{Te}_{3}$ surface. Column graph of the statistical analysis of the native defects over a total area of $500 \times 500 \mathrm{~nm}^{2}$ for all three $\mathrm{Bi}_{2} \mathrm{Te}_{3}$ crystals. The indicated doping character ( $n$-type or $p$-type) of each native defect is based on our DFT-based calculation of the PDOS spectra (see Figures SM6-SM10).

The density analysis of the native defects that occur in the top QL of the $\mathrm{Bi}_{2} \mathrm{Te}_{3}$ samples is presented in the column graph of Figure 3 (also, see Table SM1). Furthermore, from our DFT-based calculations of the projected density of states (PDOS) spectra for each native defect, the $n$-type or $p$-type doping character can be inferred (see Figures SM6-SM10). 
We find that the total amount of native defects increases slightly when the cooling rate is decreased for the Te-rich crystals and further increases for the stoichiometric $\mathrm{Bi}_{2} \mathrm{Te}_{3}$ crystal, but remains of the same order of magnitude. Interestingly, for the stoichiometric $\mathrm{Bi}_{2} \mathrm{Te}_{3}, \mathrm{~V}_{\mathrm{Te}}$ defects occuring at the Te1 sublattice are as frequent as $\mathrm{V}_{\mathrm{Te}}$ defects occurring on the Te2 sublattice, unlike what is expected when relying on the DFT-based calculations reported in literature, where $\mathrm{V}_{\mathrm{Te}}, 1$ and $\mathrm{V}_{\mathrm{Te}}, 5$ defects have lower formation energy than $\mathrm{V}_{\mathrm{Te}}, 3$ defects. 16 [24 The latter is only obeyed in the Te-rich $\mathrm{Bi}_{2} \mathrm{Te}_{3}$ crystal where $\mathrm{V}_{\mathrm{Te}}, 1$ defects have a higher density than $\mathrm{V}_{\mathrm{Te}}, 3$ defects. On the other hand, Te-rich $\mathrm{SC} \mathrm{Bi}_{2} \mathrm{Te}_{3}$ exhibits a higher density of $\mathrm{V}_{\mathrm{Te}}, 3$ defects than of $\mathrm{V}_{\mathrm{Te}}, 1$ defects. For Te-rich conditions, $\mathrm{Te}_{\mathrm{Bi}}$ defects are expected to be dominant, but in our experiments $\mathrm{Te}_{\mathrm{Bi}}$ defects were not observed and $\mathrm{Bi}_{\mathrm{Te}}$ defects occurring in the Te1 sublattice have the same probability as $V_{T e}$ defects. Based on our STM topographies, we determined that in the stoichiometric $\mathrm{Bi}_{2} \mathrm{Te}_{3}, \mathrm{Bi}_{\mathrm{Te}}$ defects occurring in the $\mathrm{Te} 2$ sublattice $\left(\mathrm{Bi}_{\mathrm{Te}}, 3\right)$ are the most frequently observed defects, but nevertheless the sample is $n$-type doped, as will be demonstrated below. Apparently, this results from the double donor character of the $\mathrm{V}_{\mathrm{Te}}$ defects compared to the single acceptor nature of $\mathrm{Bi}_{\mathrm{Te}}$ defects. $\frac{1226}{21}$ Although previous calculations report charge neutrality for $\mathrm{V}_{\mathrm{Te}}$ defects, our STM measurements and DFT-based calculations support the donor character of the $\mathrm{V}_{\mathrm{Te}}, 1, \mathrm{~V}_{\mathrm{Te}}, 3$, and $\mathrm{V}_{\mathrm{Te}}, 5$ defects. $\stackrel{23}{23}$ Furthermore, our results go along with the reported high evaporation rate of Te during growth, resulting in the fact that using a mixture with 60 at.\% of Te for the crystal growth is already sufficient to induce $n$-type doping. $\frac{19}{19}$ Evaporation of Te atoms during the crystal growth can explain the formation of Te vacancies that result in $n$-type $\mathrm{Bi}_{2} \mathrm{Te}_{3}$. In addition, the $\mathrm{V}_{\mathrm{Bi}}$ defect is not observed in our samples and hence is not identified. The difference in the density of native defects between the samples that were grown in a Te rich environment, but with different cooling rates, reveals and highlights the importance of growth kinetics, which has been observed for $\mathrm{Bi}_{2} \mathrm{Se}_{3}$ as well. $\underline{30}$

The relation between the native defects and the electronic properties of $\mathrm{Bi}_{2} \mathrm{Te}_{3}$ is unveiled by STS measurements. The normalized differential conductance $(\mathrm{d} I / \mathrm{d} V) /(I / V)$ spec- 
(a)

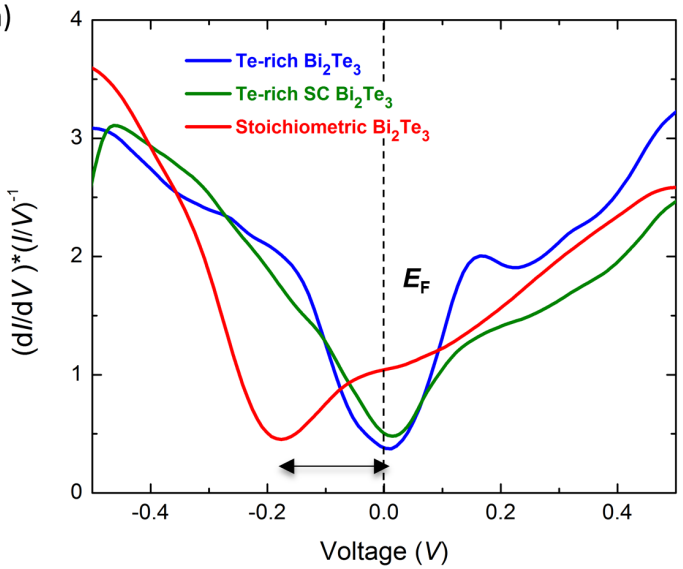

(c)

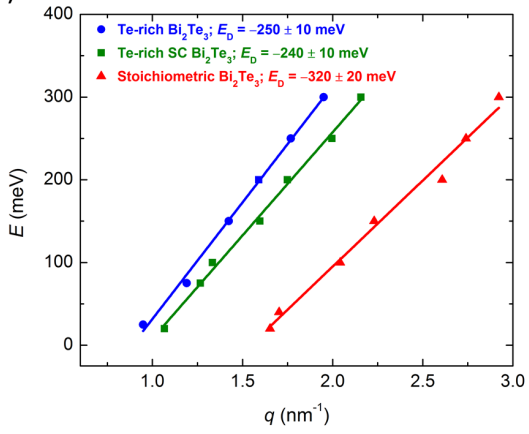

(b)

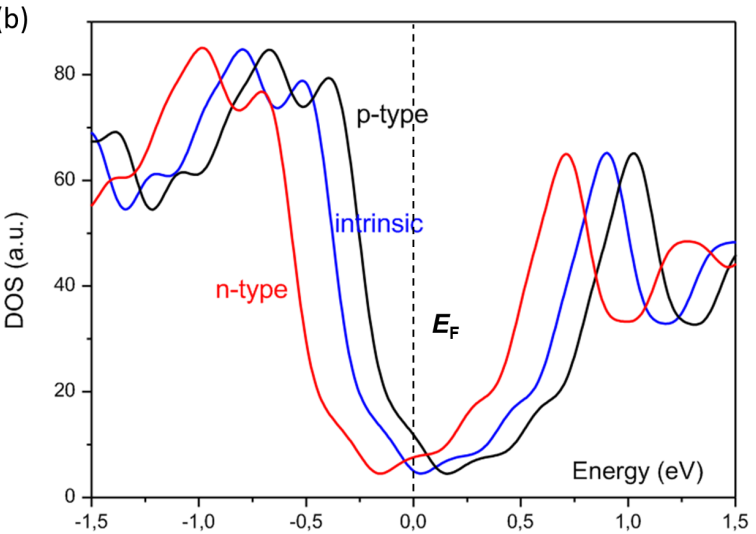

(e)

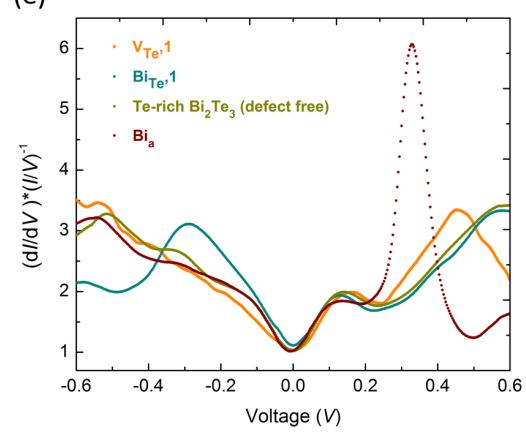

Figure 4: Surface electronic properties of $\mathrm{Bi}_{2} \mathrm{Te}_{3}$ crystals measured by STS and simulated using DFT-based calculations. (a) $(\mathrm{d} I / \mathrm{d} V) /(I / V)$ spectra (open feedback loop) recorded on the surface of the Te-rich $\mathrm{Bi}_{2} \mathrm{Te}_{3}$ (blue curve), the Te-rich $\mathrm{SC} \mathrm{Bi}_{2} \mathrm{Te}_{3}$ (green curve) and the stoichiometric $\mathrm{Bi}_{2} \mathrm{Te}_{3}$ (red curve). (b) DFT-based calculations with spin-orbit coupling of the DOS spectra for $n$-type (red curve), intrinsic (blue curve) and $p$-type (black curve) $\mathrm{Bi}_{2} \mathrm{Te}_{3}$ (15 QLs slab). (c) Energy dispersion of the surface state of the Te-rich $\mathrm{Bi}_{2} \mathrm{Te}_{3}$ (blue symbols), Te-rich $\mathrm{SC} \mathrm{Bi}_{2} \mathrm{Te}_{3}$ (green symbols) and stoichiometric $\mathrm{Bi}_{2} \mathrm{Te}_{3}$ (red symbols), including the linear fittings of the data points that allow to determine the Dirac point energies $E_{\mathrm{D}}$. (d) $1.2 \times 1.2 \mathrm{~nm}^{-2}$ FT-QPI maps corresponding to the Te-rich $\mathrm{Bi}_{2} \mathrm{Te}_{3}\left(\mathrm{~d}_{\mathrm{i}}, \mathrm{d}_{\mathrm{ii}}\right)$, the Terich $\mathrm{SC} \mathrm{Bi}_{2} \mathrm{Te}_{3}\left(\mathrm{~d}_{\mathrm{iii}}, \mathrm{d}_{\mathrm{iv}}\right)$ and the stoichiometric $\mathrm{Bi}_{2} \mathrm{Te}_{3}\left(\mathrm{~d}_{\mathrm{v}}, \mathrm{d}_{\mathrm{vi}}\right)$, acquired at the indicated voltages. (e) $(\mathrm{d} I / \mathrm{d} V) /(I / V)$ spectra (open feedback loop) recorded on top of a $\mathrm{V}_{\mathrm{Te}}$ vacancy in the $1^{\text {st }}$ atomic layer (orange points), on top of a $\mathrm{Bi}_{\mathrm{Te}}$ antisite in the $1^{\text {st }}$ atomic layer (cyan points), on a defect free area (dark yellow points) and on top of a Bi adatom (dark red points). 
tra measured at the surface of the investigated samples are proportional to the local density of states (LDOS) and are illustrated in Figure 4a. The stoichiometric $\mathrm{Bi}_{2} \mathrm{Te}_{3}$ crystal shows a minimum in the $(\mathrm{d} I / \mathrm{d} V) /(I / V)$ spectrum at $-180 \pm 5 \mathrm{meV}$ below the Fermi level $\left(E_{\mathrm{F}}=0 \mathrm{eV}\right)$, whereas the Te-rich and Te-rich $\mathrm{SC} \mathrm{Bi}_{2} \mathrm{Te}_{3}$ exhibit minima at approximately $2 \pm 5 \mathrm{meV}$ and $7 \pm 5 \mathrm{meV}$ above $E_{\mathrm{F}}$, respectively. From the $(\mathrm{d} I / \mathrm{d} V) /(I / V)$ spectrum of the stoichiometric $\mathrm{Bi}_{2} \mathrm{Te}_{3}$, it can be inferred that $E_{\mathrm{F}}$ is positioned very close to the bottom of the bulk conduction band (BCB), and hence one can consider this sample as $n$-type doped ${ }^{31}$ Moreover, it is clear that by growing $\mathrm{Bi}_{2} \mathrm{Te}_{3}$ crystals in Te rich environment $E_{\mathrm{F}}$ can be modified and positioned inside the energy gap, as illustrated in Figure 4a. The resulting doping effect in the Te-rich and Te-rich $\mathrm{SC} \mathrm{Bi}_{2} \mathrm{Te}_{3}$ crystals is compared to our DFT-based calculations of the DOS of $n$-type, intrinsic and $p$-type $\mathrm{Bi}_{2} \mathrm{Te}_{3}$ near $E_{\mathrm{F}}$, which are presented in Figure $4 \mathrm{~b}$. The qualitative agreement between the experimental data and the calculated DOS spectra suggests that the samples grown in Te-rich environment approximate intrinsic

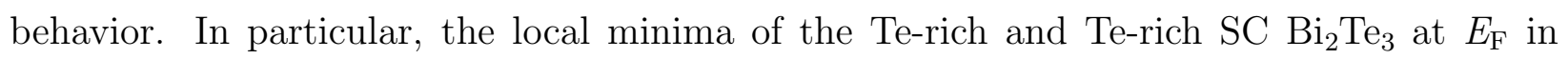
Figure 4 atch the local minimum of intrinsic $\mathrm{Bi}_{2} \mathrm{Te}_{3}$ in Figure $4 \mathrm{~b}$, while the local minimun of stoichiometric $\mathrm{Bi}_{2} \mathrm{Te}_{3}$ in Figure 4 a shifts to lower energies, corresponding to a $n$-type behavior as can be seen in Figure $4 \mathrm{~b}$. Our STS results indicate that by growing $\mathrm{Bi}_{2} \mathrm{Te}_{3}$ crystals in Te-rich environment, electronic properties approach intrinsic behavior with $E_{\mathrm{F}}$ inside the energy gap.

Next, we determine the Dirac point $\left(E_{\mathrm{D}}\right)$ for all three crystalline materials from the energy dispersion of the TSSs electrons. LDOS mapping of the local electronic structure, socalled quasiparticle interference (QPI) mapping, allows the visualization and investigation of QPI patterns created by local point defects. ${ }^{32}$ As a result of the electron interference due to the scattering of the TSSs electrons by point defects, the QPI maps reveal high periodicity, which can be analyzed in more detail by producing the Fourier-transformed (FT) images. An example of the bias-dependent FT images of the real space QPI maps is presented in Figure 44d, where the FT-QPI patterns of the measured samples are illustrated. For the 
indicated bias voltages, one can observe hexagonal patterns in the FT-QPI maps along the $\bar{\Gamma}-\overline{\mathrm{M}}$ direction. The noticeable scattering events along the $\bar{\Gamma}-\overline{\mathrm{M}}$ direction point to an increase of the wave vector length of the hexagonal geometry in the FT-QPI maps from left to right, as illustrated in Figure $4 \mathrm{~d}$, which captures the variation of the surface electronic properties of the $\mathrm{Bi}_{2} \mathrm{Te}_{3}$ crystals grown under different conditions. The prominent difference between the electronic properties of the crystals grown in Te-rich environment and the stoichiometric $\mathrm{Bi}_{2} \mathrm{Te}_{3}$ can be also observed in the energy dispersion plots in Figure 4⿻, where we demonstrate the linear energy dispersion of the TSSs determined from the biasdependent FT-QPI maps. The slope is obtained from a linear fitting of the data points to a linear energy dispersion $E(k)=E_{\mathrm{D}}+\hbar v_{\mathrm{F}} k{ }^{[3]}$ We find the Dirac point energy to shift from $E_{\mathrm{D}}=-320 \pm 20 \mathrm{meV}$ for the stoichiometric $\mathrm{Bi}_{2} \mathrm{Te}_{3}$ to $E_{\mathrm{D}}=-250 \pm 10 \mathrm{meV}$ for the Te-rich $\mathrm{Bi}_{2} \mathrm{Te}_{3}$ and to $E_{\mathrm{D}}=-240 \pm 10 \mathrm{meV}$ for the Te-rich $\mathrm{SC} \mathrm{Bi}_{2} \mathrm{Te}_{3}$. The Fermi velocity is $v_{\mathrm{F}}=5.5 \times 10^{5} \mathrm{~m} / \mathrm{s}$ (stoichiometric $\left.\mathrm{Bi}_{2} \mathrm{Te}_{3}\right), v_{\mathrm{F}}=7.4 \times 10^{5} \mathrm{~m} / \mathrm{s}\left(\right.$ Te-rich $\mathrm{Bi}_{2} \mathrm{Te}_{3}$ ) and $v_{\mathrm{F}}=6.6 \times 10^{5} \mathrm{~m} / \mathrm{s}$ (Te-rich $\mathrm{SC} \mathrm{Bi}_{2} \mathrm{Te}_{3}$ ). Therefore, our analysis of the STS results shows that a Te rich environment during growth drastically shifts the Dirac point towards higher energies.

As noted previously, the Te-rich and Te-rich $\mathrm{SC}_{\mathrm{Bi}_{2}} \mathrm{Te}_{3}$ crystals host among other native defects, Bi adatoms that are found in the topmost atomic layer. Clearly, for the Bi adatom one can observe in Figure 4 4 an electronic resonance above $E_{\mathrm{F}}$ with a pronounced increase of the LDOS at $0.33 \pm 0.05 \mathrm{eV}$. Such observation may be related to the local attractive potential exerted by the adatom and may be explained in terms of a bound state that is split off from the bottom of the $\mathrm{BCB}$ of the $\mathrm{Bi}_{2} \mathrm{Te}_{3}$. The adatom-induced resonance has not been reported for adatoms found on the $2 \mathrm{D}$ TSSs of $\mathrm{Bi}_{2} \mathrm{Te}_{3}$ but a similar observation has been reported in a non-TI system. ${ }^{34}$ Furthermore, a closer look at the LDOS near $E_{\mathrm{F}}$ of the defects occurring in the topmost atomic layer for the Te-rich $\mathrm{Bi}_{2} \mathrm{Te}_{3}$ (see Figure 4 4 ), reveals an increase of the LDOS (defect state at a bias voltage of $-0.30 \pm 0.05 \mathrm{eV}$ ) at the valence band for the case of the $\mathrm{Bi}_{\mathrm{Te}}, 1$ antisite defect, implying an acceptor character for this type of 
defect. On the other hand, the electronic signature of the $\mathrm{V}_{\mathrm{Te}}, 1$ defects reveals a pronounced increase of the LDOS (defect state at a bias voltage of $0.45 \pm 0.05 \mathrm{eV}$ ) at the conduction band of the Te-rich $\mathrm{Bi}_{2} \mathrm{Te}_{3}$, pointing to a donor character.

\section{Conclusions}

We successfully identified native point defects that exist in $\mathrm{TI}_{\mathrm{Bi}_{2}} \mathrm{Te}_{3}$ crystals by using STM/STS experiments and DFT-based calculations. The investigated samples are $\mathrm{Bi}_{2} \mathrm{Te}_{3}$ crystals grown under stoichiometric conditions and under Te-rich conditions (two different cooling rates for the latter). Our results demonstrate that the resulting density of native defects depends not only on the Bi and Te atomic concentrations during growth, but on the growth kinetics as well. Furthermore, our STS measurements reveal that a Te rich environment during growth drastically shifts the Dirac point towards higher energies and yields $E_{\mathrm{F}}$ inside the energy gap. As a result, Te-rich $\mathrm{Bi}_{2} \mathrm{Te}_{3}$ crystals approximate intrinsic behavior, while $\mathrm{Bi}_{2} \mathrm{Te}_{3}$ crystals grown under stoichiometric conditions result in $n$-type doping with $E_{\mathrm{F}}$ lying at the bottom of the BCB. Our results establish a bias-dependent STM signature of the $\mathrm{Bi}_{2} \mathrm{Te}_{3}$ native defects and underline the significance of both thermodynamics and kinetics during growth conditions, which can promote the synthesis of intrinsic TI samples and may offer an appropriate route for improving the functional properties of TI materials.

\section{Experimental and computational methods}

\section{Sample preparation}

The $\mathrm{Bi}_{2} \mathrm{Te}_{3}$ crystals referred to as stoichiometric were prepared by heating the stoichiometric mixture (i.e., with the exact $2 / 3$ ratio of the atomic concentrations) of high-purity $\mathrm{Bi}$ and Te powders ( $5 \mathrm{~N}$, both from Alfa Aesar) in sealed quartz ampules, at a speed of $20^{\circ} \mathrm{C} / \mathrm{h}$, to $850^{\circ} \mathrm{C}$ and kept at this high temperature for 3 days. The mixture was stirred continuously 
to avoid segregation of the ingredients. After slowly cooling to $550{ }^{\circ} \mathrm{C}$ in 9 days, the material was annealed at $550{ }^{\circ} \mathrm{C}$ for 5 days. The sample was naturally cooled down to room temperature from $550{ }^{\circ} \mathrm{C}$. Te-rich and Te-rich $\mathrm{SC} \mathrm{Bi}_{2} \mathrm{Te}_{3}$ crystals $(6 \mathrm{~N})$ were purchased from the 2D Semiconductors company. $\stackrel{35}{\text { B5 }}$ Both samples were grown by the flux method in a Te-rich environment (with a Te excess of about 0.1 at.\% Te). The Te-rich $\mathrm{SC} \mathrm{Bi}_{2} \mathrm{Te}_{3}$ sample was grown with a three times slower cooling rate. All of the probed $\mathrm{Bi}_{2} \mathrm{Te}_{3}$ samples were in situ cleaved with a ultra-high vacuum compatible carbon tape at base pressure in the $10^{-11} \mathrm{mbar}$ range.

\section{STM experiments}

STM/STS experiments were carried out in a ultra-high vacuum, low-temperature STM (Omicron Nanotechnology) operated at $4.5 \mathrm{~K}$ (base pressure in the $10^{-11}$ mbar range). $(\mathrm{d} I / \mathrm{d} V) /(I / V)$ spectra and $\mathrm{d} I / \mathrm{d} V$ maps (referred to as LDOS maps) were obtained by lock-in detection at $800 \mathrm{~Hz}$ with open and closed feedback loops, respectively. STM data were acquired using mechanically cut PtIr (10\% Ir) STM tips and polycrystalline W tips that were electrochemically etched and cleaned in situ by thermal treatment. All bias voltages mentioned are with respect to the sample, and the STM tip was virtually grounded. STM data were analyzed using the Nanotec WSxM software. $\underline{36}$

\section{DFT-based calculations}

The structural and electronic properties of $\mathrm{Bi}_{2} \mathrm{Te}_{3}$ surface and native defects were analyzed using first-principle DFT-based calculations ${ }^{[37}$ within the Perdew-Burke-Ernzerhof ${ }^{[38}$ generalized gradient approximation $\underline{39}$ exchange-correlation energy functional with and without taking into account the effects of spin-orbit coupling (SOC) $\stackrel{40}{ }$ Calculations were performed using the SIESTA code, ${ }^{41}$ which relies on the expansion of the Kohn-Sham orbitals using a linear combination of pseudoatomic orbitals. The core electrons were implicitly treated by using norm-conserving Troullier-Martins pseudopotentials. ${ }^{42}$ A mesh cutoff of 400 Ry for the 
grid integration and a split double-zeta basis set were used in all calculations. Integrals over the Brillouin zone were summed on a $9 \times 9 \times 1\left(1 \times 1 \times 1\right.$ for the supercells) Monkhorst-Pack ${ }^{43}$ mesh, ensuring convergence of the self-consistent field iteration process until the changes of total energy were below $0.1 \mathrm{meV}$. The $\mathrm{Bi}_{2} \mathrm{Te}_{3}$ surface was simulated by a slab with $(1 \times 1)$ surface unit cells consisting of one to $18 \mathrm{QL}(\mathrm{s})$ (depending on the type of calculations) separated by a vacuum space of $26.7 \AA$ along the $z$-direction of the slabs. Investigations of the electronic properties such as band structure and total DOS were carried out with SOC using a one to $18 \mathrm{QL}(\mathrm{s})(1 \times 1) \mathrm{Bi}_{2} \mathrm{Te}_{3}$ slab. To model the $\mathrm{Bi}_{2} \mathrm{Te}_{3}$ surface structure, we used the supercell approach without taking into account SOC. In the triple QLs $(7 \times 7)$ and in the septuple QLs $(6 \times 6)$ supercells a single native defect was introduced and the equilibrium configurations of the $\mathrm{Bi}_{2} \mathrm{Te}_{3}$ system were determined using conjugate gradient geometry optimization. The convergences of the atomic geometry relaxation were carried out until the absolute values of all atomic forces acting on the released atoms were smaller than $5 \mathrm{meV} / \AA$. The calculated geometries of the relaxed septuple QLs $(6 \times 6)$ supercell were used for the PDOS analysis, while the relaxed triple QLs $(7 \times 7)$ supercell were transferred to larger $(11 \times 11)$ or $(13 \times 13)$ supercells (double QLs), for which we calculated the final electronic properties in detail. The simulated STM images were generated using the Tersoff-Hamann approximation ${ }^{44 \mid 45}$ at a distance from $2 \AA$ up to $8 \AA$ above the surface. In order to evaluate the surface wave function in the vacuum region up to $z=10 \AA$ above the surface, we relied on the 2D FT of the wave functions in combination with spatial extrapolation. $\underline{46}+\underline{48}$

\section{Supporting Information}

The Supporting Information is available on the ACS Publications website.

Bias-dependent STM topographies of $\mathrm{Bi}_{2} \mathrm{Te}_{3}$ native defects, bias-dependent simulated STM images of $\mathrm{Bi}_{2} \mathrm{Te}_{3}$ native defects not observed in the experiments, additional STM topographies presenting the observed mobility of Bi adatoms, table presenting the densities 
of the native defects, additional DFT-based calculations of the doping character of the native defects, and the DFT-based calculated electronic band structure of $\mathrm{Bi}_{2} \mathrm{Te}_{3}$ for 1 up to 18 QLs.

\section{Acknowledgement}

The work at KU Leuven has been supported by the Research Foundation - Flanders (FWO, Belgium) and by the KU Leuven C1 program (project C12/18/006). The research in Moscow has been supported by the computing facilities of the M. V. Lomonosov Moscow State University (MSU) Research Computing Center. The work at Nanjing University has been supported by the National Key Projects for the Basic Research of China (grants 2013CB922103 and 2011CB922103), the National Natural Science Foundation of China (grants 91421109, 11134005, 11522432, and 11274003), the Natural Science Foundation of Jiangsu Province (grant BK20130054), and the Fundamental Research Funds for the Central Universities.

\section{References}

1. Kane, C. L.; Mele, E. J. Z2 Topological Order and the Quantum Spin Hall Effect. Phys. Rev. Lett. 2005, 95, 146802.

2. Bernevig, B. A.; Zhang, S. C. Quantum Spin Hall Effect. Phys. Rev. Lett. 2006, 96, 106802.

3. Bernevig, B. A.; Hughes, T. L.; Zhang, S. C. Quantum Spin Hall Effect and Topological Phase Transition in HgTe Quantum Wells. Science 2006, 314, 1757-1761.

4. König, M.; Wiedmann, S.; Brüne, C.; Roth, A.; Buhmann, H.; Molenkamp, L. W.; Qi, X. L.; Zhang, S. C. Quantum Spin Hall Insulator State in HgTe Quantum Wells. Science 2007, 318, 766-770. 
5. Zhang, H.; Liu, C. X.; Qi, X. L.; Dai, X.; Fang, Z.; Zhang, S. C. Topological Insulators in $\mathrm{Bi}_{2} \mathrm{Se}_{3}, \mathrm{Bi}_{2} \mathrm{Te}_{3}$ and $\mathrm{Sb}_{2} \mathrm{Te}_{3}$ with a Single Dirac Cone on the Surface. Nat. Phys. 2009, $5,438-442$.

6. Xia, Y.; Qian, D.; Hsieh, D.; Wray, L.; Pal, A.; Lin, H.; Bansil, A.; Grauer, D.; Hor, Y. S.; Cava, R. J.; Hasan, M. Z. Observation of a Large-Gap Topological-Insulator Class with a Single Dirac Cone on the Surface. Nat. Phys. 2009, 5, 398-402.

7. Chen, Y. L.; Analytis, J. G.; Chu, J. H.; Liu, Z. K.; Mo, S. K.; Qi, X. L.; Zhang, H. J.; Lu, P. H.; Dai, X.; Fang, Z.; Zhang, S. C.; Fisher, I. R.; Hussain, Z.; Shen, Z. X. Experimental Realization of a Three-Dimensional Topological Insulator, $\mathrm{Bi}_{2} \mathrm{Te}_{3}$. Science 2009, 325, 178-181.

8. Hsieh, D.; Xia, Y.; Qian, D.; Wray, L.; Dil, J. H.; Meier, F.; Osterwalder, J.; Patthey, L.; Checkelsky, J. G.; Ong, N. P.; Fedorov, A. V.; Lin, H.; Bansil, A.; Grauer, D.; Hor, Y. S.; Cava, R. J.; Hasan, M. Z. A Tunable Topological Insulator in the Spin Helical Dirac Transport Regime. Nature 2009, 460, 1101-1105.

9. Hsieh, D.; Xia, Y.; Wray, L.; Qian, D.; Pal, A.; Dil, J. H.; Osterwalder, J.; Meier, F.; Bihlmayer, G.; Kane, C. L.; Hor, Y. S.; Cava, R. J.; Hasan, M. Z. Observation of Unconventional Quantum Spin Textures in Topological Insulators. Science 2009, 323, 919-922.

10. Roushan, P.; Seo, J.; Parker, C. V.; Hor, Y. S.; Hsieh, D.; Qian, D.; Richardella, A.; Hasan, M. Z.; Cava, R. J.; Yazdani, A. Topological Surface States Protected from Backscattering by Chiral Spin Texture. Nature 2009, 460, 1106-1109.

11. Fu, L.; Kane, C. L. Superconducting Proximity Effect and Majorana Fermions at the Surface of a Topological Insulator. Phys. Rev. Lett. 2008, 100, 096407.

12. Wang, L. L.; Huang, M.; Thimmaiah, S.; Alam, A.; Bud'Ko, S. L.; Kaminski, A.; Lo- 
grasso, T. A.; Canfield, P.; Johnson, D. D. Native Defects in Tetradymite $\mathrm{Bi}_{2}\left(\mathrm{Te}_{\mathrm{x}} \mathrm{Se}_{3-\mathrm{x}}\right)$ Topological Insulators. Phys. Rev. B. 2013, 87, 125303.

13. Qu, D. X.; Hor, Y. S.; Xiong, J.; Cava, R. J.; Ong, N. P. Quantum Oscillations and Hall Anomaly of Surface States in the Topological Insulator $\mathrm{Bi}_{2} \mathrm{Te}_{3}$. Science 2010, 329, $821-824$.

14. Checkelsky, J. G.; Hor, Y. S.; Liu, M. H.; Qu, D. X.; Cava, R. J.; Ong, N. P. Quantum Interference in Macroscopic Crystals of Nonmetallic $\mathrm{Bi}_{2} \mathrm{Se}_{3}$. Phys. Rev.Lett. 2009, 103, 246601.

15. Butch, N. P.; Kirshenbaum, K.; Syers, P.; Sushkov, A. B.; Jenkins, G. S.; Drew, H. D.; Paglione, J. Strong Surface Scattering in Ultrahigh-Mobility $\mathrm{Bi}_{2} \mathrm{Se}_{3}$ Topological Insulator Crystals. Phys. Rev. B 2010, 81, 241301.

16. Hashibon, A.; Elsässer, C. First-Principles Density Functional Theory Study of Native Point Defects in $\mathrm{Bi}_{2} \mathrm{Te}_{3}$. Phys. Rev. B 2011, 84, 14-16.

17. Satterthwaite, C. B.; Ure, R. W. Electrical and Thermal Properties of $\mathrm{Bi}_{2} \mathrm{Te}_{3}$. Phys. Rev. 1957, 108, 1164-1170.

18. Miller, G. R.; Li, C. Y. Evidence for the Existence of Antistructure Defects in Bismuth Telluride by Density Measurements. J. Phys. Chem. Solids 1965, 26, 173-177.

19. Da Silva, L. W.; Kaviany, M.; Uher, C. Thermoelectric Performance of Films in the Bismuth-Tellurium and Antimony-Tellurium Systems. J. Appl. Phys 2005, 97, 114903.

20. Nakajima, S. The Crystal Structure of $\mathrm{Bi}_{2} \mathrm{Te}_{3-x} \mathrm{Se}_{\mathrm{x}}$. J. Phys. Chem. Solids 1963, 24, $479-485$.

21. Mishra, S. K.; Satpathy, S.; Jepsen, O. Electronic Structure and Thermoelectric Properties of Bismuth Telluride and Bismuth Selenide. J. Phys. Condens. Matter 1997, 9, $461-470$. 
22. Pecheur, P.; Toussaint, G. Electronic Structure and Bonding in Bismuth Telluride. Phys. Lett. A 1989, 135, 223-226.

23. Wang, G.; Zhu, X. G.; Sun, Y. Y.; Li, Y. Y.; Zhang, T.; Wen, J.; Chen, X.; He, K.; Wang, L. L.; Ma, X. C.; Jia, J. F.; Zhang, S. B.; Xue, Q. K. Topological Insulator Thin Films of $\mathrm{Bi}_{2} \mathrm{Te}_{3}$ With Controlled Electronic Structure. Adv. Mater. 2011, 23, 2929-2932.

24. Scanlon, D. O.; King, P. D.; Singh, R. P.; De La Torre, A.; Walker, S. M. K.; Balakrishnan, G.; Baumberger, F.; Catlow, C. R. Controlling Bulk Conductivity in Topological Insulators: Key Role of Anti-Site Defects. Adv. Mater. 2012, 24, 2154-2158.

25. West, D.; Sun, Y. Y.; Wang, H.; Bang, J.; Zhang, S. B. Native Defects in SecondGeneration Topological Insulators: Effect of Spin-Orbit Interaction on $\mathrm{Bi}_{2} \mathrm{Se}_{3}$. Phys. Rev. B 2012, 86, 121201.

26. Pecheur, P.; Toussaint, G. Tight-Binding Studies of Crystal Stability and Defects in $\mathrm{Bi}_{2} \mathrm{Te}_{3}$. J. Phys. Chem. Solids 1994, 55, 327-338.

27. Reifsnyder Hickey, D.; Mkhoyan, K. A. Atomic-Resolution Analytical Scanning Transmission Electron Microscopy of Topological Insulators with a Layered Tetradymite Structure. APL Materials 2020, 8, 070902.

28. Callaert, C.; Bercx, M.; Lamoen, D.; Hadermann, J. Interstitial Defects in the van der Waals Gap of $\mathrm{Bi}_{2} \mathrm{Se}_{3}$. Acta Crystallogr., Sect. B: Struct. Sci., Cryst. Eng. Mater. 2019, $75,717-732$.

29. Jiang, Y.; Sun, Y. Y.; Chen, M.; Wang, Y.; Li, Z.; Song, C.; He, K.; Wang, L.; Chen, X.; Xue, Q. K.; Ma, X.; Zhang, S. B. Fermi-Level Tuning of Epitaxial $\mathrm{Sb}_{2} \mathrm{Te}_{3}$ Thin Films on Graphene by Regulating Intrinsic Defects and Substrate Transfer Doping. Phys. Rev. Lett. 2012, 108, 066809. 
30. Dai, J.; West, D.; Wang, X.; Wang, Y.; Kwok, D.; Cheong, S. W.; Zhang, S. B.; Wu, W. Toward the Intrinsic Limit of the Topological Insulator $\mathrm{Bi}_{2} \mathrm{Se}_{3}$. Phys. Rev. Lett. 2016, 117, 106401.

31. Alpichshev, Z.; Analytis, J. G.; Chu, J. H.; Fisher, I. R.; Chen, Y. L.; Shen, Z. X.; Fang, A.; Kapitulnik, A. STM Imaging of Electronic Waves on the Surface of $\mathrm{Bi}_{2} \mathrm{Te}_{3}$ : Topologically Protected Surface States and Hexagonal Warping Effects. Phys. Rev. Lett. 2010, $104,016401$.

32. Lee, W. C.; Wu, C.; Arovas, D. P.; Zhang, S. C. Quasiparticle Interference on the Surface of the Topological Insulator $\mathrm{Bi}_{2} \mathrm{Te}_{3}$. Phys. Rev. B 2009, 80, 245439.

33. Zhang, T.; Cheng, P.; Chen, X.; Jia, J. F.; Ma, X.; He, K.; Wang, L.; Zhang, H.; Dai, X.; Fang, Z.; Xie, X.; Xue, Q. K. Experimental Demonstration of Topological Surface States Protected by Time-Reversal Symmetry. Phys. Rev. Lett. 2009, 103, 266803.

34. Schouteden, K.; Debehets, J.; Muzychenko, D.; Li, Z.; Seo, J. W.; Van Haesendonck, C. Adsorption of Te Atoms on $\mathrm{Au}(111)$ and the Emergence of an Adatom-Induced Bound State. J. Phys. Condens. Matter 2017, 29, 125001.

35. 2D Semiconductors. https://www.2dsemiconductors.com/bismuth-telluride-bi2te3/ (accessed October 8, 2020).

36. Horcas, I.; Fernández, R.; Gómez-Rodríguez, J. M.; Colchero, J.; Gómez-Herrero, J.; Baro, A. M. WSXM: A Software for Scanning Probe Microscopy and a Tool for Nanotechnology. Rev. Sci. Instrum 2007, 78, 013705.

37. Kohn, W.; Sham, L. J. Self-Consistent Equations Including Exchange and Correlation Effects. Phys. Rev. 1965, 140, A1133-A1138.

38. Perdew, J. P.; Burke, K.; Ernzerhof, M. Generalized Gradient Approximation Made Simple. Phys. Rev. Lett. 1996, 77, 3865-3868. 
39. Perdew, J. P.; Yue, W. Accurate and Simple Density Functional for the Electronic Exchange Energy: Generalized Gradient Approximation. Phys. Rev. B 1986, 33, 88008802 .

40. Fernández-Seivane, L.; Oliveira, M. A.; Sanvito, S.; Ferrer, J. On-Site Approximation for Spin-Orbit Coupling in Linear Combination of Atomic Orbitals Density Functional Methods. J. Phys. Condens. Matter 2006, 18, 7999-8013.

41. Soler, J. M.; Artacho, E.; Gale, J. D.; García, A.; Junquera, J.; Ordejón, P.; SánchezPortal, D. The SIESTA Method for ab Initio Order-N Materials Simulation. J. Phys. Condens. Matter 2002, 14, 2745-2779.

42. Troullier, N.; Martins, J. L. Efficient Pseudopotentials for Plane-Wave Calculations. Phys. Rev. B 1991, 43, 1993-2006.

43. Monkhorst, H. J.; Pack, J. D. Special Points for Brillouin-Zone Integrations. Phys. Rev. $B$ 1976, 13, 5188-5192.

44. Tersoff, J.; Hamann, D. R. Theory and Application for the Scanning Tunneling Microscope. Phys. Rev. Lett. 1983, 50, 1998-2001.

45. Tersoff, J. and Hamann, D. R. Theory of the Scanning Tunneling Microscope. Phys. Rev. $B$ 1985, 31, 805-813.

46. Muzychenko, D. A.; Schouteden, K.; Houssa, M.; Savinov, S. V.; Van Haesendonck, C. Noninvasive Embedding of Single Co Atoms in Ge(111)2×1 Surfaces. Phys. Rev. B 2012, 85,125412 .

47. Muzychenko, D. A.; Schouteden, K.; Van Haesendonck, C. Electronic and Atomic Structure of Co/Ge Nanoislands on the Ge(111) Surface. Phys. Rev. B 2013, 88, 195436.

48. Rohlfing, M.; Temirov, R.; Tautz, F. S. Adsorption Structure and Scanning Tunneling 
Data of a Prototype Organic-Inorganic Interface: PTCDA on Ag(111). Phys. Rev. B 2007, 76, 115421. 


\section{Graphical TOC Entry}

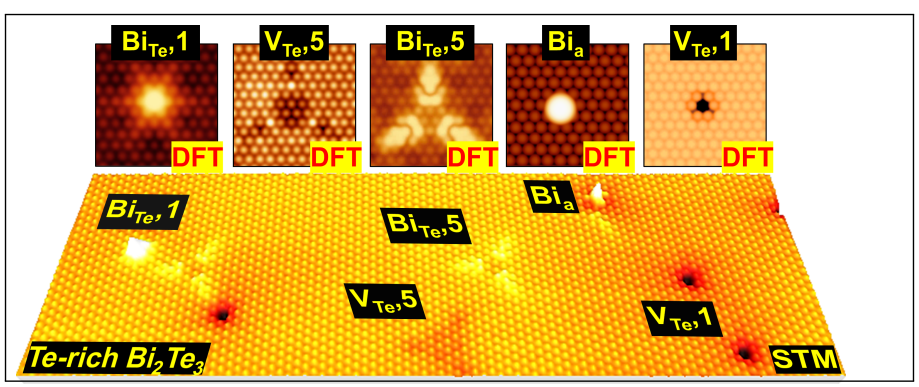

